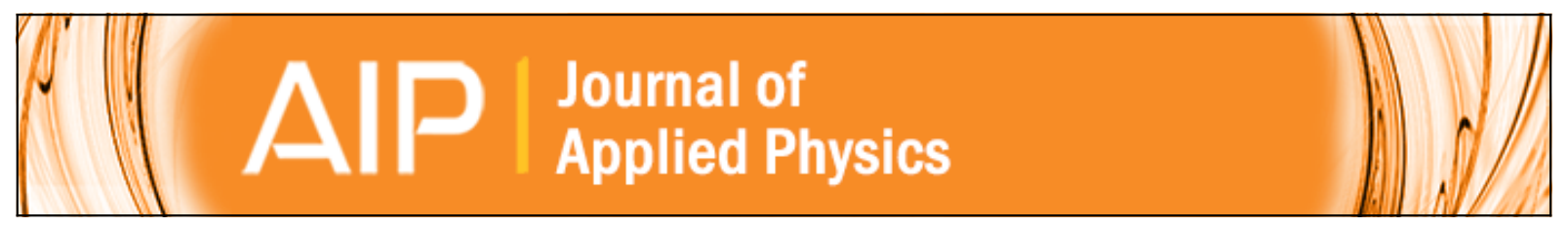

\title{
Nanoindentation-induced phase transformation in relaxed and unrelaxed ion-implanted amorphous germanium
}

D. J. Oliver, J. E. Bradby, S. Ruffell, J. S. Williams, and P. Munroe

Citation: Journal of Applied Physics 106, 093509 (2009); doi: 10.1063/1.3255999

View online: http://dx.doi.org/10.1063/1.3255999

View Table of Contents: http://scitation.aip.org/content/aip/journal/jap/106/9?ver=pdfcov

Published by the AIP Publishing

\section{Articles you may be interested in}

Effect of hydrogen on nanoindentation-induced phase transformations in amorphous silicon

J. Appl. Phys. 106, 123511 (2009); 10.1063/1.3267853

Annealing of nanoindentation-induced high pressure crystalline phases created in crystalline and amorphous silicon

J. Appl. Phys. 105, 093513 (2009); 10.1063/1.3124366

Effect of oxygen concentration on nanoindentation-induced phase transformations in ion-implanted amorphous silicon

J. Appl. Phys. 105, 083520 (2009); 10.1063/1.3097752

Phase transformations induced by spherical indentation in ion-implanted amorphous silicon

J. Appl. Phys. 100, 013520 (2006); 10.1063/1.2210767

Indentation-induced crystallization and phase transformation of amorphous germanium

J. Appl. Phys. 96, 1464 (2004); 10.1063/1.1766414
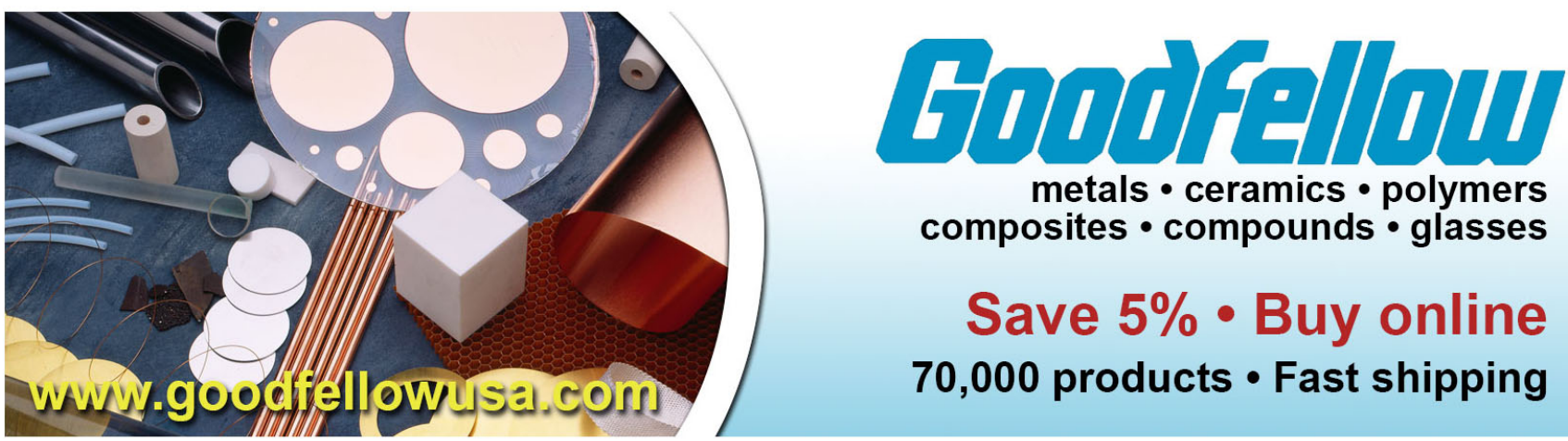


\title{
Nanoindentation-induced phase transformation in relaxed and unrelaxed ion-implanted amorphous germanium
}

\author{
D. J. Oliver, ${ }^{1, a)}$ J. E. Bradby, ${ }^{2}$ S. Ruffell, ${ }^{2}$ J. S. Williams, ${ }^{2}$ and P. Munroe ${ }^{3}$ \\ ${ }^{1}$ Department of Physics, McGill University, Montréal, Québec H3A 2T8, Canada \\ ${ }^{2}$ Department of Electronic Materials Engineering, Research School of Physics and Engineering, The \\ Australian National University, Canberra, Australian Capital Territory 0200, Australia \\ ${ }^{3}$ Electron Microscope Unit, University of New South Wales, Sydney, New South Wales 2052, Australia
}

(Received 26 August 2009; accepted 29 September 2009; published online 6 November 2009)

\begin{abstract}
We have investigated nanoindentation-induced plastic deformation in amorphous germanium (a-Ge) prepared by high-energy self-ion implantation. Using cross-sectional transmission electron microscopy, micro-Raman spectroscopy, and force-displacement curve analysis, we find strong evidence for a pressure-induced metallic phase transformation during indentation. Crystalline diamond-cubic Ge-I is observed in residual indents. Relaxed and unrelaxed structural states of a-Ge exhibit similar behavior on loading, but transform at different pressures on unloading. Both forms are markedly softer mechanically than crystalline Ge. These results assist in furthering the understanding of the intriguing phenomenon known as "explosive crystallization." (c) 2009 American Institute of Physics. [doi:10.1063/1.3255999]
\end{abstract}

\section{INTRODUCTION}

Amorphous solids have been the subject of intensive research for over 70 years. ${ }^{1}$ These materials are distinguished from crystalline solids by the absence of long-range order. The amorphous elemental semiconductors, germanium (a$\mathrm{Ge})$ and silicon (a-Si), provide ideal model amorphous systems that can be directly compared with their crystalline counterparts.

Under appropriate conditions, self-ion implantation can be used to form amorphous Ge in a high-purity, porosity-free form. ${ }^{2}$ When this as-implanted amorphous phase is annealed at temperatures below the crystallization temperature, structural relaxation is observed, resulting in a drop in free energy $^{3}$ and reduced disorder (bond length and bond angle distortion). ${ }^{4-7}$ The structural differences between these two states (i.e., as-implanted and "relaxed" a-Ge) are not completely understood, although a common point of view is that the relaxed state approaches the condition of a continuous random network (CRN) with fourfold coordination throughout, whereas the as-implanted "unrelaxed" state features a higher concentration of dangling bonds and greater bond distortion. ${ }^{8}$

When subjected to high pressure in a diamond-anvil cell (DAC), a-Ge has been observed to undergo a structural transformation to a metallic phase at $\sim 6-7 \mathrm{GPa},{ }^{9-13}$ marked by a large drop in resistivity, ${ }^{9}$ volume decrease, ${ }^{10,11}$ changes in optical properties, ${ }^{10,11}$ and change in the atomic structure. ${ }^{11,12}$ There is some disagreement as to the phase formed at $6-7$ GPa: some investigators found it to be the $\beta$-tin structure of $\mathrm{Ge},{ }^{11,12}$ but others describe it as a high-density amorphous phase (hda-Ge). ${ }^{9,13,14}$ After pressure release from $7 \mathrm{GPa}$, the resulting phase is polycrystalline Ge-I. ${ }^{9-12}$ An in situ $\mathrm{X}$-ray diffraction (XRD) study by Imai et al. ${ }^{12}$ found that the metallic phase on unloading first transformed to a body-

${ }^{a)}$ Electronic mail: oliverd@physics.mcgill.ca. centered cubic phase with 8 atoms per unit cell (bc-8 Ge or Ge-IV), then to Ge-I. This sequence of transformations is illustrated in Fig. 1.

The response of crystalline $\mathrm{Ge}$ (c-Ge) to nanoindentation with a sharp diamond tip has previously been investigated. Although there are indications that a pressure-induced phase transformation takes place in some circumstances, ${ }^{15-18}$ under most standard loading conditions it appears that the dominant deformation mechanisms are shear-induced dislocation slip and twinning. ${ }^{19-22}$

The nanoindentation response of amorphous $\mathrm{Si}(\mathrm{a}-\mathrm{Si})$ has also been studied in some detail. ${ }^{23-26}$ The preferred deformation mechanism is found to depend on the method of preparation. Relaxed a-Si deforms via a pressure-induced phase transformation similar to crystalline $\mathrm{Si}$ (c-Si). In contrast, unrelaxed a-Si shows a tendency to deform via shear flow, except under confinement. Both forms are softer than c-Si, particularly unrelaxed a-Si.

To date, however, to the authors' knowledge there has only been one nanoindentation study on amorphous Ge (aGe), by Patriarche $e t$ al. ${ }^{27}$ This study was performed on a-Ge films prepared by low-temperature electron-beam evaporation onto a GaAs substrate. Ge-I was observed in residual impressions by transmission electron microscopy (TEM) and Raman spectroscopy after indentation. The structural state of

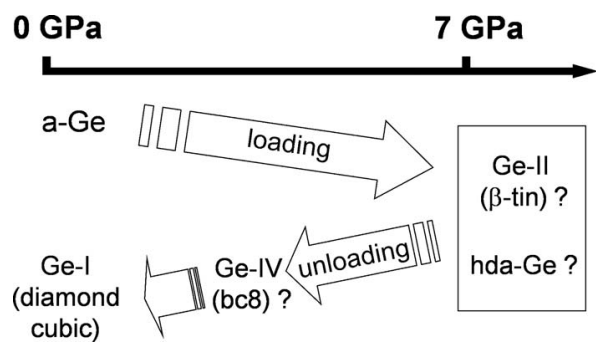

FIG. 1. Schematic showing the phase transformations undergone by a-Ge upon loading to high pressure and after pressure release. 
the as-deposited a-Ge film was not examined by Patriarche $e t$ al. However, it is known that deposited amorphous films can contain significant porosity ${ }^{28,29}$ and impurities. ${ }^{30}$ Void fractions of up to $10 \mathrm{vol} \%$ have been observed for deposited a-Ge films, ${ }^{28,29}$ which could significantly influence deformation behavior. $^{31}$ Additionally, nanoindentation forcedisplacement curves were not shown in the study by Patriarche et al.

This paper examines the nanoindentation response of a-Ge prepared by high-energy self-ion implantation, i.e., films containing low levels of impurities and no porosity, in both as-implanted and relaxed states.

\section{EXPERIMENTAL DETAILS}

Amorphous layers were obtained by ion implantation using the ANU 1.7 MV NEC Pelletron tandem accelerator. Implants were carried out at room temperature. Undoped Ge(100) wafers (Wafer World, West Palm Beach) were implanted with $800 \mathrm{keV}$ energy $\mathrm{Ge}$ ions to fluences of 3 $\times 10^{14}$ and $3 \times 10^{15}$ ions $\mathrm{cm}^{-2}$. Raman spectroscopy confirmed that the layers were fully amorphous up to the surface. Ion damage profiles simulated with the SRIM software package $^{32}$ predicted an amorphous layer thickness of $\sim 700 \mathrm{~nm}$, which was confirmed by TEM.

After implantation, some specimens were annealed under argon flow in a quartz tube furnace for $30 \mathrm{~min}$ at $250{ }^{\circ} \mathrm{C}$. Annealing at this temperature relaxes the structure ${ }^{3,6,7}$ of ionimplanted a-Ge without inducing significant recrystallization. ${ }^{33}$ Throughout this paper, the term relaxed will be used to refer to annealed specimens and the term unrelaxed to describe as-implanted unannealed specimens.

Amorphized specimens were indented with the UMIS2000 nanoindenter (CSIRO, Australia). A spherical indenter tip with radius $\sim 4.3 \mu \mathrm{m}$ was used. Maximum loads of up to $100 \mathrm{mN}$ were applied, with loading and unloading rates of $\mathrm{d} P / \mathrm{d} h \approx 1 \mathrm{mN} \mathrm{s}^{-1}$. Hardness values were calculated from tests to $50 \mathrm{mN}$ with the spherical tip, using the Oliver and Pharr method. ${ }^{34}$

Micro-Raman spectroscopy was carried out using a Renishaw 2000 Raman microscope operating at $632.8 \mathrm{~nm}$ to characterize the end phases obtained in indents. The laser power was kept below $100 \mu \mathrm{W}$ to avoid annealing any sensitive metastable phases. ${ }^{22}$

The level of structural disorder in relaxed and unrelaxed amorphous specimens was assessed by measuring the linewidth of the transverse optical (TO)-like Raman band of a-Ge centered at $\sim 270 \mathrm{~cm}^{-1}$. Broadening of this band is proportional to the bond angle distribution in the amorphous network, ${ }^{5}$ giving a measure of disorder and deviation from the ideal CRN structure. The linewidth was obtained from Raman spectra by measuring the half-width at halfmaximum on the high-frequency side of the band, to avoid the contribution on the low-frequency side from the transverse acoustic peak. ${ }^{5}$

To examine indentation-induced deformation and phase transformation in detail, TEM cross sections were prepared of selected indents by a focused ion beam (FIB) "lift-out" technique $^{35}$ using a xT Nova NanoLab 200 dual-beam FIB.
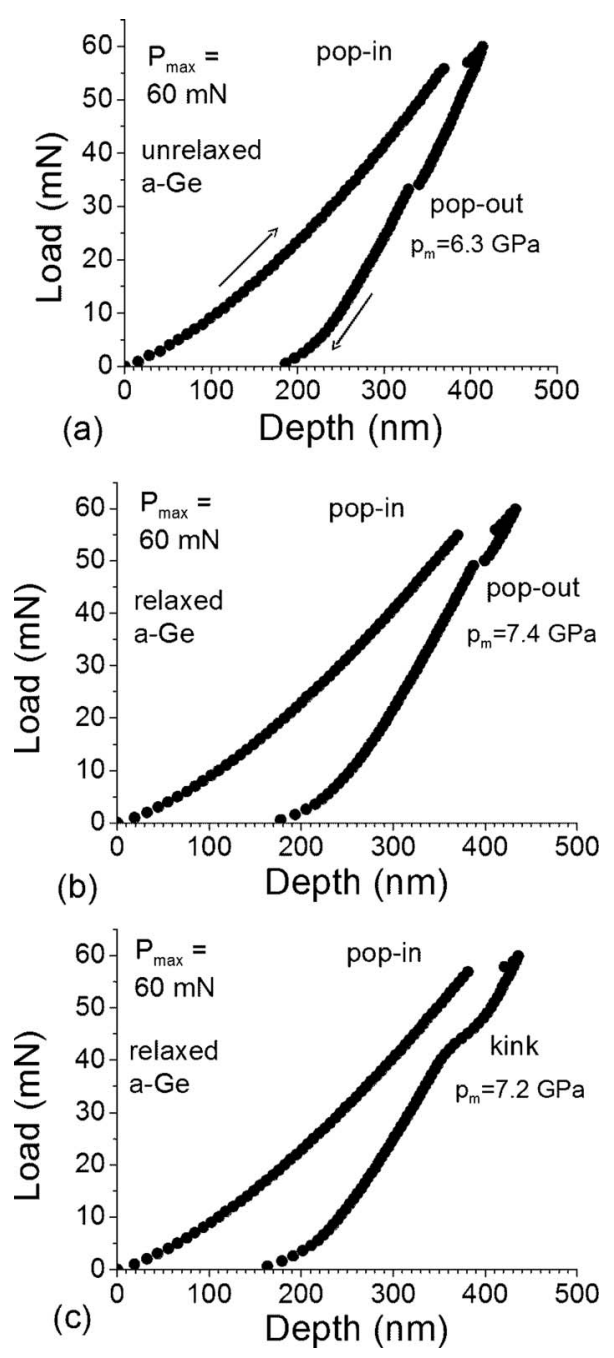

FIG. 2. $60 \mathrm{mN}$ maximum load $P$ - $h$ curves for indents in a-Ge samples, (a) unrelaxed and (b), (c) relaxed. Curves [(a) and (b)] feature a pop-out on unloading, curve (c) features a kink.

Specimens were coated with gold prior to insertion, and once inside the FIB the regions of interest were coated with platinum to prevent ion beam-induced damage. Cross sections were imaged with a Philips CM 300 TEM operating at 300 $\mathrm{kV}$.

\section{RESULTS}

Force-displacement $(P-h)$ curves for both unrelaxed and relaxed a-Ge are shown in Fig. 2. Except where otherwise indicated, results presented throughout the paper will be from the $3 \times 10^{15}$ ions $\mathrm{cm}^{-2}$ fluence samples. $P-h$ curves consistently featured a single pop-in on loading at a welldefined load. The $60 \mathrm{mN}$ maximum load indents shown in Fig. 2 feature either a pop-out (displacement burst) on unloading, or a kink (slope discontinuity) on unloading. Both pop-outs and kinks have been observed when $\mathrm{Si}$ is indented and are caused in that case by the sudden transformation of a discrete volume of material from a high-density phase to a low-density phase. ${ }^{36}$ Notably, the pop-out for the unrelaxed a-Ge film in Fig. 2(a) occurs at a load about $15 \mathrm{mN}$ lower than the pop-out for the relaxed film in Fig. 2(b). 


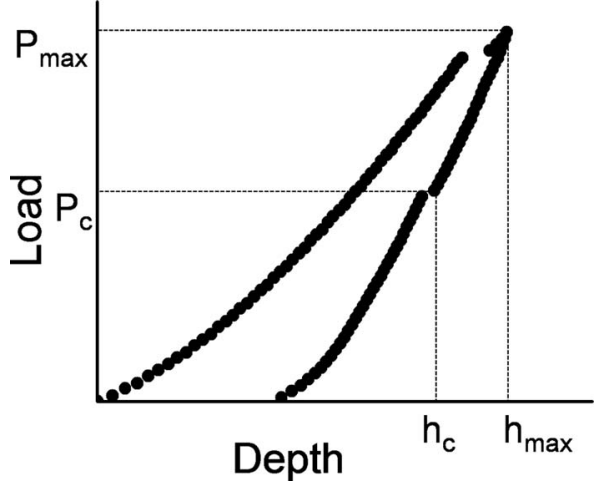

FIG. 3. The mean pressure under the indenter prior to the pop-out can be calculated from the measured force and depth values shown on the curve.

The force and depth at the point when pop-out or kinking occurs can be translated into a pressure under the indenter using the method of Field and Swain. ${ }^{37,38}$ The contact radius at maximum load $\left(a_{\max }\right)$ is first calculated according to the relationship $a_{\max }=\sqrt{2 R h_{p}-h_{p}^{2}}$, where $R$ is the indenting tip radius and $h_{p}$ is the plastic depth given by the formula

$$
h_{p}=\frac{1}{2} \frac{\left.\left(h_{\max }+h_{c}\right)\left(P_{\max } / P_{c}\right)^{2 / 3}\right)-2 h_{\max }}{\left.P_{\max } / P_{c}\right)^{2 / 3}-1},
$$

where $P_{\max }$ and $h_{\max }$ are the maximum force and depth, and $P_{c}$ and $h_{c}$ are the force and depth at the point of pop-out or kinking, as measured from the data (Fig. 3). The contact radius $a_{c}$ just before the pop-out or kink is then given by

$$
a_{c}=a_{\max }\left(\frac{P_{c}}{P_{\max }}\right)^{1 / 3} \text {. }
$$

From this, the mean pressure can be determined, $p_{m}=P_{c} / \pi a_{c}^{2}$.

The pressure at pop-out/kink initiation was calculated for $60 \mathrm{mN}$ maximum load indents. From eight tests in unrelaxed a-Ge, the average pressure at pop-out or kinking was $6.2 \pm .2 \mathrm{GPa}$. From 12 tests in relaxed a-Ge, the average was $7.3 \pm 0.1 \mathrm{GPa}$.

For indents to $60 \mathrm{mN}$ in unrelaxed a-Ge, $22 \%$ of unload curves featured a pop-out, $7 \%$ featured a kink, and $71 \%$ were featureless from 28 tests. For $60 \mathrm{mN}$ indents in relaxed a-Ge, $7 \%$ of unload curves featured a pop-out, $36 \%$ featured a kink, and $57 \%$ were featureless, again from 28 tests.

Indents to maximum loads below the pop-in load generally did not feature pop-outs or kinks at all on unloading.

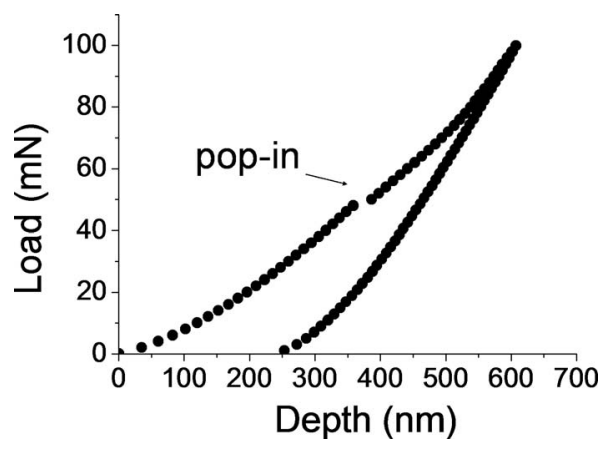

FIG. 4. $P$ - $h$ curve to $100 \mathrm{mN}$ in unrelaxed a-Ge sample.

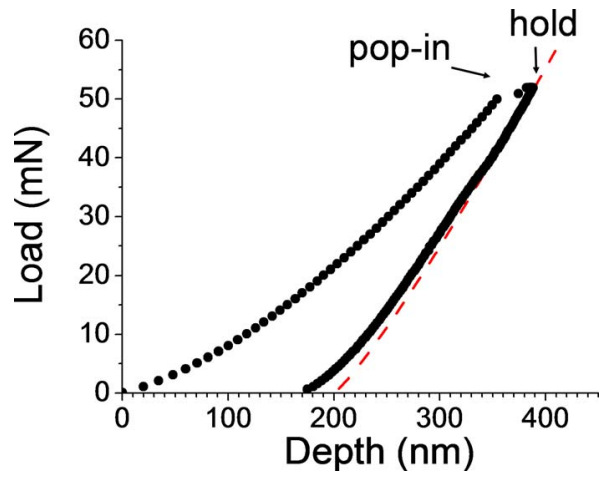

FIG. 5. (Color online) $P$ - $h$ curve to $50 \mathrm{mN}$, with a $30 \mathrm{~s}$ hold at maximum load, in unrelaxed a-Ge sample. The dashed line is a guide to the eyes to visualize slope changes on unloading.

Tests to a maximum load well above the pop-in load also typically had featureless unload curves, as shown for the 100 $\mathrm{mN}$ load indent in Fig. 4. Finally, tests made to a load just above the pop-in threshold, but with a holding time of $30 \mathrm{~s}$ at maximum load, also tended not to feature a pop-out or kink on unloading, as shown in Fig. 5. Note the unload curve in Fig. 5 is not entirely featureless but appears to feature slight slope changes (very weak kinking) indicative of minor departure from ideal elastic-plastic behavior.

The hardness values measured for the a-Ge specimens are shown in Fig. 6. Each plotted hardness value represents an average from a number of tests, with error bars representing scatter within the averaged values (one standard deviation). The hardness is $\sim 7.5 \mathrm{GPa}$ for the $3 \times 10^{14}$ and 3 $\times 10^{15}$ ions $\mathrm{cm}^{-2}$ fluence specimens, compared with the hardness for $\mathrm{c}-\mathrm{Ge}$ of $\sim 10.7 \mathrm{GPa}$. The hardness values of relaxed and unrelaxed specimens appear to be approximately the same.

Raman spectra from the amorphous layers with no indent present featured only the broad band centered at $\sim 270 \mathrm{~cm}^{-1}$ characteristic of a-Ge, with no Ge-I peak. For indents in these specimens to loads of $50-60 \mathrm{mN}$, the Raman spectrum varied: some indents contained only a-Ge, while other indents contained crystalline Ge-I in addition to a-Ge. There was no obvious correlation between the phase within the indent and the $P$ - $h$ curve features corresponding to the indent. Indents to loads of $100 \mathrm{mN}$ consistently featured the Ge-I peak, sometimes with little or no a-Ge. Some representative spectra are shown in Fig. 7.

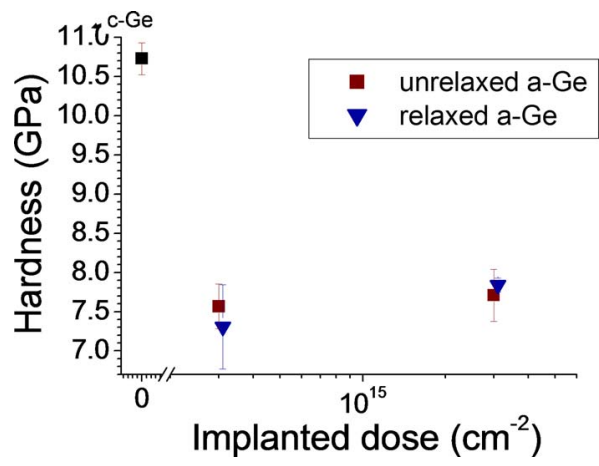

FIG. 6. (Color online) Hardness vs implanted fluence for unrelaxed and relaxed a-Ge samples. 

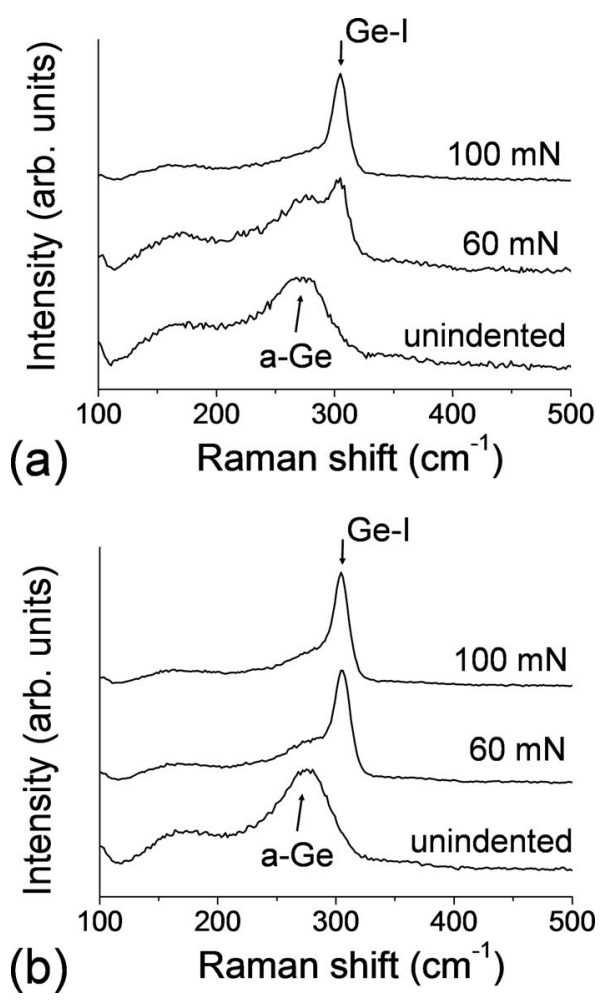

FIG. 7. Raman spectra taken from indents in a-Ge, (a) unrelaxed and (b) relaxed. Undeformed a-Ge spectra shown for comparison.

Figure 8 shows the linewidth of the TO-like Raman band for unrelaxed and relaxed specimens. Notably, the linewidth is reduced significantly for the relaxed specimens, confirming that a reduction in disorder and bond distortion takes place during annealing. ${ }^{5-7}$

TEM of an indent in an unrelaxed specimen is shown in Fig. 9(a). Outside the indent, the implanted layer shows the uniform contrast characteristic of pure amorphous material, which is confirmed by the selected area diffraction pattern (SADP) [Fig. 9(b)]. Directly beneath the indent, however, there is a large recrystallized region. The SADP from this region shows that it contains polycrystalline Ge-I and perhaps some a-Ge [Fig. 9(c)]. No additional reflections are present in the SADP, indicating that there are no other crystalline phases present. The shape of the recrystallized zone is roughly hemispherical and does not extend into the underlying crystalline material.

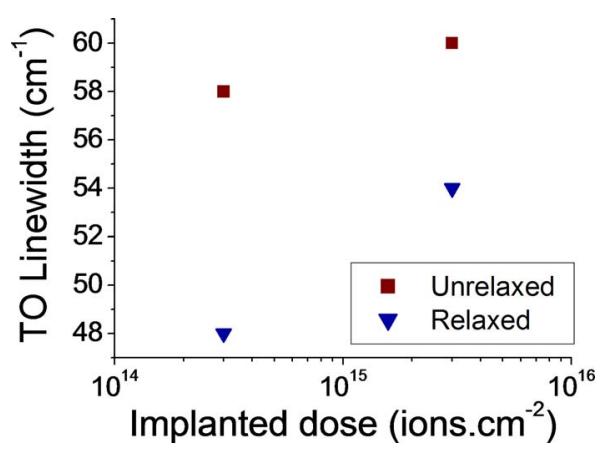

FIG. 8. (Color online) TO linewidth measured from Raman spectra for unindented specimens plotted vs implantation dose, for both unrelaxed (asimplanted) and relaxed $\left(250^{\circ} \mathrm{C}\right.$ annealed) specimens.

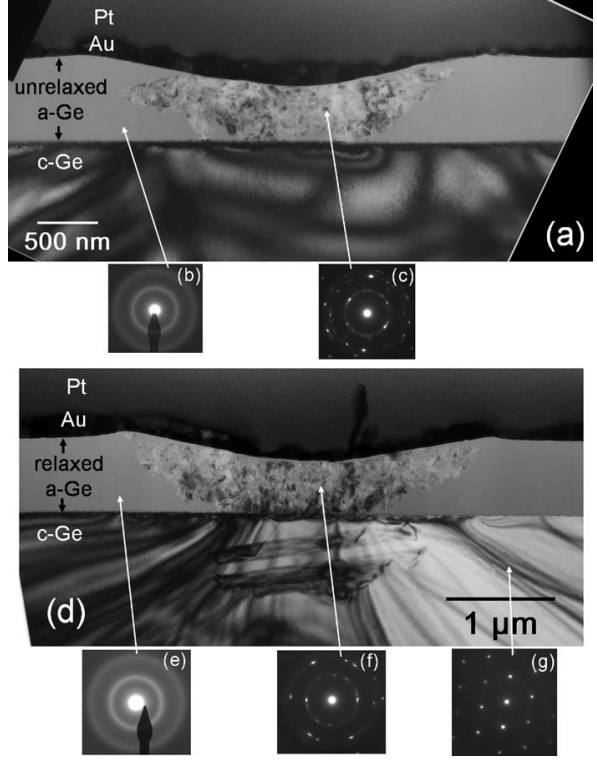

FIG. 9. (a) Bright-field (BF) cross-sectional TEM micrograph of an indent to $60 \mathrm{mN}$ in unrelaxed a-Ge. (b) SADP from the amorphous layer, away from the indent. (c) SADP from recrystallized region under indent. (d) BF cross-sectional TEM micrograph of an indent to $60 \mathrm{mN}$ in relaxed a-Ge. (e) SADP from the amorphous layer away from the indent. (f) SADP from recrystallized region under indent. (g) SADP from pristine underlying c-Ge away from indent and amorphous layer.

Figure 9(d) shows TEM of an indent in a relaxed specimen with SADPs shown in Figs. 9(e)-9(g) from the unindented amorphous layer, indented and crystallized region, and underlying pristine crystal, respectively. The deformation pattern is similar to that of the unrelaxed specimen and similarly the hemispherical crystallized zone contains polycrystalline Ge-I.

\section{DISCUSSION}

To aid in understanding the indentation response of a-Ge, it is instructive to consider the sequence of transformations of the material in a DAC. As outlined in Sec. I and Fig. 1 , a-Ge transforms to a metallic phase at $\sim 6-7 \mathrm{GPa}$, either to $\beta$-tin $\mathrm{Ge}$, or to hda-Ge according to different studies. ${ }^{9-14}$ An in situ XRD study by Imai et al. ${ }^{12}$ found that, on unloading, the metallic phase first transforms to bc- $8 \mathrm{Ge}$ (Ge-IV), which is unstable at room temperature and ambient pressure and reverts to Ge-I. Other authors likewise reported Ge-I present after unloading to ambient pressure with no reports of intermediate phases being present.

By contrast $\beta$-tin Ge formed by compression of Ge-I typically transforms to a simple tetragonal phase with 12 atoms per unit cell (st-12 Ge or Ge-III) on pressure release. ${ }^{39,40}$ This implies that the high-pressure phase obtained from compression of a-Ge is different in some way to that obtained with Ge-I. One explanation is that Ge-I transforms under pressure to $\beta$-tin Ge-II whereas a-Ge converts to hda-Ge. ${ }^{13,14}$ Supporting this view, Shimomura et al. ${ }^{9}$ observed a second metal-to-metal transition by electrical resistance measurements at $\sim 10 \mathrm{GPa}$, which may be a hda-Ge to Ge-II conversion. They obtained st-12 Ge after pressure release from $\sim 10 \mathrm{GPa}$, whereas Ge-I was obtained after pressure release from $\sim 7 \mathrm{GPa}$. However, other DAC studies 
employing in situ XRD have shown evidence that the phase transformation at $\sim 6-7 \mathrm{GPa}$ is an a-Ge to $\beta$-tin transformation. ${ }^{11}$ Hence, understanding of this phenomenon from the literature is inconsistent. It should be noted that DAC studies have all been carried out on deposited a-Ge, which as already mentioned can contain significant impurities and inhomogeneity, ${ }^{29,30}$ possibly contributing to the discrepancies across different studies.

Nonetheless, the finding that Ge-I is observed within residual indents in a-Ge is totally consistent with a highpressure phase transformation taking place during indentation despite the fact that the phase transformation sequence is not clear. Furthermore, the hardness measured for both relaxed and unrelaxed a-Ge is around 7.5 GPa (Fig. 6), close to the transformation pressure for a-Ge from most DAC studies. $^{9-13}$ Pop-out and kink features indicative of a high-density $\rightarrow$ low-density phase transformation ${ }^{38,41,42}$ were observed in our study on unloading, at a calculated pressure of $\sim 6.2 \mathrm{GPa}$ for the unrelaxed a-Ge film and $\sim 7.2 \mathrm{GPa}$ for the relaxed a-Ge film. By comparison, the unloading transformation in DAC studies has been reported to occur at 4-5 GPa. ${ }^{11,12}$ This suggests that the deposited a-Ge used in DAC studies is closer to the unrelaxed ion-implanted a-Ge. Consequently, it would be worthwhile to investigate the highpressure response of ion-implanted a-Ge under DAC compression to see if a similar behavior is observed.

The hardness values for relaxed and unrelaxed a-Ge specimens are approximately the same, despite the differences in bonding and structure observed (Fig. 8). This is perhaps understandable if the critical transition pressure for phase transformation is the governing factor of hardness in each case, and if it is assumed that the transition pressure is the same ( $\sim 7 \mathrm{GPa})$ for both relaxed and unrelaxed a-Ge. By contrast, relaxed and unrelaxed ion-implanted a-Si states do differ in hardness, with unrelaxed a-Si slightly softer. This has been linked to the fact these a-Si states exhibit differing responses to indentation, with phase transformation observed for relaxed a-Si and shear-induced deformation for unrelaxed a-Si. ${ }^{25}$

It should be noted that for $\mathrm{Si}$, when the scale of deformation is large relative to the thickness of the a-Si film, the harder $\mathrm{c}-\mathrm{Si}$ substrate confines further plastic flow and phase transformation is observed even for unrelaxed $\mathrm{a}-\mathrm{Si}^{26}$ The c-Ge substrate might be playing a similar confinement role in the current study. The greater degree of crystallization (Ge-I in the residual indent) observed for the $100 \mathrm{mN}$ indents (Fig. 7) hints at this. If the underlying crystal substrate was influencing the phase transformation, the crystalline material could conceivably act as a template to promote the formation of single-crystal Ge-I on unloading. We note that the diffraction pattern from the transformed zone [Figs. 9(c) and 9(f)] is one of polycrystalline Ge-I, suggesting instead a random nucleation and growth mode of formation. Further experiments on thicker a-Ge films would be needed to further understand the role of the substrate in seeding crystallization.

The absence of a pop-out or kink for indents to higher maximum loads (Fig. 4) and indents with a hold period (Fig. 5 ) is intriguing. For these indents Ge-I was still observed by Raman spectroscopy (Fig. 7) and TEM, indicating that a phase transformation is still taking place. The absence of a discrete feature implies that the phase transformation is occurring in a continuous fashion throughout unloading, rather than a sudden onset at a critical load. One possible explanation is the presence of a small amount of the lower-density Ge-I or Ge-IV phases prior to unloading, which would remove the initial nucleation barrier to phase transformation responsible for the pop-out. In this scenario, during the longer loading time or hold time a small amount of lowerdensity phase could form, acting as a nucleus upon unloading. Although formation of a lower-density phase under load seems counterintuitive, it is actually supported by the DAC observation of Shimomura et al. ${ }^{9}$ who observed by resistivity measurements that some of the high-pressure metallic phase reverted to a high-resistance (semiconducting) phase over time while holding at constant pressure. This is another point of departure from the phase transformation behavior observed during indentation of a-Si and c-Si.

Finally, our observations on the behavior of a-Ge under indentation appear to be relevant to the notable phenomenon known as "explosive crystallization" of a-Ge. This refers to a process whereby the recrystallization of a small volume of a-Ge can trigger a runaway reaction that recrystallizes a large region of amorphous material. ${ }^{43}$ This occurs because the a-Ge to Ge-I transformation is an exothermic reaction, since Ge-I has a lower free energy than a-Ge. Under appropriate conditions the a-Ge $\rightarrow$ Ge-I transformation of a small region of amorphous film releases sufficient heat to recrystallize adjoining material, which in turn releases more heat to stimulate further crystallization, and so on in a somewhat selfsustaining reaction. The original paper to report explosive crystallization in a-Ge found that it could be triggered by, among various methods that included pulsed laser heating, "pricking with a sharp point," process. In this latter case it seems plausible that the initial trigger for crystallization occurred via a high-pressure phase transformation. However, explosive crystallization beyond the indenter contact area was not observed in the present study. This may have been as a result of the confining (compressive) effect of the indenter, the relatively slow unloading rate compared with a sudden impact with a sharp point, or the fact that heat loss to the underlying c-Ge bulk sample was in our case too high. Heat dissipation could be suppressed by using thicker amorphous films and elevated temperatures $^{44,45}$ and ultrafast unloading could be used to achieve a regime in which indentation-triggered explosive crystallization is possible. A thorough study of indentationinduced explosive crystallization of a-Ge would indeed complement extensive existing research into laser-induced explosive crystallization. ${ }^{43-46}$

\section{CONCLUSION}

We have investigated the indentation-induced deformation in ion-implanted a-Ge films. For both structurally relaxed and unrelaxed specimens, phase transformation was observed as the dominant deformation mechanism. Phase transformation dominates in a-Ge despite the fact that shear mechanisms dominate in nanoindented c-Ge, and the local 
atomic bonding in the amorphous and crystalline forms is substantially similar. The lower pressure required for phase transformation for a-Ge relative to $\mathrm{c}-\mathrm{Ge}(\sim 7 \mathrm{GPa}$ compared with $\sim 11 \mathrm{GPa}$ ) appears to make it a more favorable deformation pathway. The similarity between relaxed and unrelaxed a-Ge film behavior is intriguing in comparison with the differing response for such a-Si states, but a confining effect of the substrate cannot be ruled out in our a-Ge study. The observed sequence of transformations in nanoindented a-Ge is consistent with the behavior of a-Ge in DAC tests. The ability to locally transform a-Ge to Ge-I by nanoindentation may prove to be useful for electronic and optoelectronic applications.

\section{ACKNOWLEDGMENTS}

We thank the Australian Research Council for funding support.

${ }^{1}$ W. H. Zachariasen, J. Am. Chem. Soc. 54, 3841 (1932).

${ }^{2}$ J. S. Williams, Rep. Prog. Phys. 49, 491 (1986).

${ }^{3}$ E. P. Donovan, F. Spaepen, D. Turnbull, J. M. Poate, and D. C. Jacobson, J. Appl. Phys. 57, 1795 (1985).

${ }^{4}$ M. A. Paesler, D. E. Sayers, R. Tsu, and J. Gonzalez-Hernandez, Phys. Rev. B 28, 4550 (1983).

${ }^{5}$ R. Tsu, J. Gonzalez-Hernandez, and F. H. Pollak, Solid State Commun. 54, 447 (1985)

${ }^{6}$ J. Fortner and J. S. Lannin, Phys. Rev. B 37, 10154 (1988).

${ }^{7}$ I. D. Desnica-Frankovic, K. Furic, U. V. Desnica, M. C. Ridgway, and C. J. Glover, Nucl. Instrum. Methods Phys. Res. B 178, 192 (2001).

${ }^{8}$ D. E. Polk and D. S. Boudreau, Phys. Rev. Lett. 31, 92 (1973).

${ }^{9}$ O. Shimomura, S. Minomura, N. Sakai, K. Asaumi, K. Tamura, J. Fukushim, and H. Endo, Philos. Mag. 29, 547 (1974).

${ }^{10}$ K. Tanaka, Solid State Commun. 76, 213 (1990).

${ }^{11}$ K. Tanaka, Phys. Rev. B 43, 4302 (1991).

${ }^{12}$ M. Imai, T. Mitamura, K. Yaoita, and K. Tsuji, High Press. Res. 15, 167 (1996).

${ }^{13}$ E. Principi, A. Di Cicco, F. Decremps, A. Polian, S. De Panfilis, and A Filipponi, Phys. Rev. B 69, 201201 (2004).

${ }^{14}$ M. Durandurdu and D. Drabold, Phys. Rev. B 66, 041201 (2002).

${ }^{15}$ G. M. Pharr, W. C. Oliver, R. F. Cook, P. D. Kirchner, M. C. Kroll, T. R. Dinger, and D. R. Clarke, J. Mater. Res. 7, 961 (1992).

${ }^{16}$ A. Kailer, K. G. Nickel, and Y. G. Gogotsi, J. Raman Spectrosc. 30, 939 (1999).

${ }^{17}$ S. J. Lloyd, J. M. Molina-Aldareguia, and W. J. Clegg, J. Mater. Res. 16, 3347 (2001).

${ }^{18}$ D. Oliver, B. Lawn, R. Cook, M. Reitsma, J. Bradby, J. Williams, and P.
Munroe, J. Mater. Res. 23, 297 (2008).

${ }^{19}$ Y. G. Gogotsi, V. Domnich, S. N. Dub, A. Kailer, and K. G. Nickel, J. Mater. Res. 15, 871 (2000).

${ }^{20}$ J. E. Bradby, J. S. Williams, J. Wong-Leung, M. V. Swain, and P. Munroe, Appl. Phys. Lett. 80, 2651 (2002).

${ }^{21}$ J. Jang, M. J. Lance, S. Wen, and G. M. Pharr, Appl. Phys. Lett. 86, 131907 (2005)

${ }^{22}$ D. J. Oliver, J. E. Bradby, J. S. Williams, M. V. Swain, and P. Munroe, J. Appl. Phys. 101, 043524 (2007).

${ }^{23}$ J. S. Williams, Y. Chen, J. Wong-Leung, A. Kerr, and M. V. Swain, J. Mater. Res. 14, 2338 (1999).

${ }^{24}$ M. M. Khayyat, G. K. Banini, D. G. Hasko, and M. M. Chaudhri, J. Phys. D 36, 1300 (2003).

${ }^{25}$ B. Haberl, J. E. Bradby, M. V. Swain, J. S. Williams, and P. Munroe, Appl. Phys. Lett. 85, 5559 (2004).

${ }^{26}$ B. Haberl, J. E. Bradby, S. Ruffell, J. S. Williams, and P. Munroe, J. Appl. Phys. 100, 013520 (2006).

${ }^{27}$ G. Patriarche, E. Le Bourhis, M. M. O. Khayyat, and M. M. Chaudhri, J. Appl. Phys. 96, 1464 (2004).

${ }^{28}$ J. E. Yehoda, B. Yang, K. Vedam, and R. Messier, J. Vac. Sci. Technol. A 6, 1631 (1988).

${ }^{29}$ J. C. G. de Sande, C. N. Afonso, J. L. Escudero, R. Serna, F. Catalina, and E. Bernabéu, Appl. Opt. 31, 6133 (1992).

${ }^{30}$ G. Schirmer, A. Duparré, W. Heerdegen, H.-J. Kühn, A. Lehmann, W. Richter, B. Schröter, E. Hacker, and J. Meyer, Phys. Status Solidi A 124, 199 (1991).

${ }^{31}$ S.-H. Jhi, S. G. Louie, M. L. Cohen, and J. Ihm, Phys. Rev. Lett. 86, 3348 (2001).

${ }^{32}$ J. F. Ziegler, www.srim.org.

${ }^{33}$ L. Csepregi, R. P. Kullen, J. W. Mayer, and T. W. Sigmon, Solid State Commun. 21, 1019 (1977).

${ }^{34}$ W. Oliver and G. Pharr, J. Mater. Res. 7, 1564 (1992).

${ }^{35}$ R. M. Langford and A. K. Petford-Long, J. Vac. Sci. Technol. A 19, 2186 (2001).

${ }^{36}$ T. Juliano, Y. G. Gogotsi, and V. Domnich, J. Mater. Res. 18, 1192 (2003).

${ }^{37}$ J. S. Field and M. V. Swain, J. Mater. Res. 8, 297 (1993).

${ }^{38}$ E. R. Weppelmann, J. S. Field, and M. V. Swain, J. Mater. Res. 8, 830 (1993).

${ }^{39}$ C. S. Menoni, J. Z. Hu, and I. L. Spain, Phys. Rev. B 34, 362 (1986).

${ }^{40}$ R. J. Nelmes, M. I. McMahon, N. G. Wright, D. R. Allan, and J. S. Loveday, Phys. Rev. B 48, 9883 (1993).

${ }^{41}$ V. Domnich, Y. Gogotsi, and S. N. Dub, Appl. Phys. Lett. 76, 2214 (2000).

${ }^{42}$ J. E. Bradby, J. S. Williams, J. Wong-Leung, M. V. Swain, and P. Munroe, Appl. Phys. Lett. 77, 3749 (2000).

${ }^{43}$ H. J. Leamy, W. L. Brown, G. K. Celler, G. Foti, G. H. Gilmer, and J. C. C. Fan, Appl. Phys. Lett. 38, 137 (1981).

${ }^{44}$ T. Takamori, R. Roy, and R. Messier, Appl. Phys. Lett. 20, 201 (1972).

${ }^{45}$ C. Grigoropoulos, M. Rogers, S. H. Ko, A. A. Golovin, and B. J. Matkowsky, Phys. Rev. B 73, 184125 (2006).

${ }^{46}$ D. Bensahel and G. Auvert, Mater. Res. Soc. Symp. Proc. 13, 165 (1983). 